\title{
Factores asociados a la elección de psiquiatría como especialidad en internos de medicina de dos universidades privadas del Perú
}

\author{
Factors associated with choosing psychiatry among medical interns in two private universities in Peru \\ Lucy Perla Gulliana Cedillo Ramírez ${ }^{1, a}$, Lucy Elena Correa López ${ }^{1, b}$, Jhony Alberto De La Cruz Vargas ${ }^{1, c}$
}

RESUMEN

Objetivo: Determinar los factores asociados a la elección de psiquiatría como especialidad médica en internos de medicina de dos universidades privadas del Perú. Material y métodos: Estudio transversal realizado en internos de medicina de las universidades Ricardo Palma y San Juan Bautista. Se les administró tres instrumentos: El Inventario de Personalidad - Eysenck para adultos (EPI), la Escala de Valoración del Proceso Enseñanza - Aprendizaje, y el Cuestionario General de Salud Mental de Goldberg (QHG-12), para el estudio de las variables personalidad, percepción del proceso enseñanza - aprendizaje del curso de psiquiatría y psicopatología respectivamente. Se utilizó la prueba estadística $\mathrm{X}^{2}$ cuadrado para determinar la existencia de asociación entre las variables. Resultados: De los 223 internos de medicina estudiados, 35 (15,7\%) manifestaron que elegirán psiquiatría como especialidad. La elección de psiquiatría como especialidad se asoció con: personalidad sanguínea $(\mathrm{p}=0,001)$; extroversión $(\mathrm{p}=0,02)$ y ser emocionalmente estable $(\mathrm{p}=0,03)$. Por otro lado, el estudio no evidenció asociación significativa entre las variables sexo, parientes con carreras afines al área de la salud mental y psicopatología con la elección de psiquiatría. Conclusiones: Existe asociación estadísticamente significativa entre el tipo de personalidad y la elección de psiquiatría. Los internos de medicina que les interesa especializarse en psiquiatría, por lo general tienen una personalidad tipo sanguínea, además son extrovertidos y emocionalmente estables.

PALABRAS CLAVE: Psiquiatría, enseñanza, aprendizaje, personalidad, especialidad médica (Fuente: DeCS BIREME).

\section{SUMMARY}

Objective: To determine the factors associated with choosing psychiatry as a specialty among medical interns in two private universities in Peru. Methods: A cross-sectional study was carried-out among medical interns of Ricardo Palma and San Juan Bautista Universities. Three instruments were applied: personality inventory-Eysenck for adults, scale to evaluate the teaching-learning process and the Goldberg's general survey of mental health to evaluate personality, perception of the teaching-learning process of the psychiatry and psychology courses. The X2 test was used to determine associations. Results: out of 233 medical interns evaluated, $35(15.7 \%)$ said that would elect psychiatry as a specialty. The election of psychiatry was associated with a blood personality ( $\mathrm{p}=0.001)$; extroversion $(\mathrm{p}=0.02)$ and being emotionally stable $(\mathrm{p}=0.039)$. On the other hand, the study did not reveal and association with sex, relatives with specialties closed to mental health and psychopathology. Conclusions: there

\footnotetext{
Instituto de Investigación de Ciencias Biomédicas (INICIB), Facultad de Medicina Humana, Universidad Ricardo Palma. Lima Perú.

a Médico Cirujano de la Universidad Ricardo Palma.

b Coordinadora de Economía de la Salud, INICIB.

c Doctor en Medicina, Maestría en Investigación Clínica.
} 
is an association between type of personality and choosing psychiatry as a) specialty. Medical interns interested in becoming psychiatrist have a blood type of personality, are extroverted and emotionally stable.

KEYWORDS: Psychiatric, teaching, learning, personality, medical specialty. (Source: MeSH NLM).

\section{INTRODUCCIÓN}

Se ha descrito que la elección de especialidad médica es un proceso que está determinado por múltiples factores tales como las características demográficas, necesidades y valores del estudiante, percepción de las especialidades, exposición de los estudiantes a las distintas disciplinas médicas durante los años clínicos, entre otros (1). Asimismo, la influencia de la personalidad en la elección vocacional de las distintas especialidades en los estudiantes de medicina, ha interesado a muchos autores a nivel mundial $(2,3)$.

De manera particular, diferentes autores en el mundo han planteado factores para la elección de psiquiatría. En España, una investigación evidenció que los estudiantes de medicina que eligen psiquiatría tienen una personalidad definida y diferenciada del resto de estudiantes que optaron por otras especialidades médicas (4).

En Latinoamérica, un estudio realizado en Chile mostró que de un total de 65 estudiantes de medicina que optaron por alguna especialidad médica, quienes escogieron psiquiatría eran más introvertidos, reflexivos e intuitivos, y con mayor tendencia al pensamiento abstracto (3).

Respecto al sexo y la elección de psiquiatría, Monleon $\mathrm{P}$ et al. (5), concluyeron que entre los estudiantes de medicina existen diferencias significativas en sus preferencias vocacionales según el sexo; de acuerdo a esto, de un total de 120 estudiantes, entre varones y mujeres, que eligieron psiquiatría, el 63,3\% fueron mujeres. Por otro lado, la experiencia en el curso de psiquiatría durante los años académicos de la carrera de medicina, proveen una visión más realista de la psiquiatría, lo cual contribuye a que más estudiantes de medicina se interesen en seguir la especialidad posteriormente $(6,7)$.

En el Perú, Alarcón (8) en un estudio que buscaba explicar la asociación de la personalidad con la elección de las distintas especialidades médicas, concluyó: "Los rasgos de personalidad varían de acuerdo a la especialidad preferida, es decir que cada especialidad albergaría personalidades similares o afines". Por su parte, Perales (9) sostiene que la forma en que se lleva el curso de psiquiatría en la universidad contribuye para persuadir al estudiante sobre las bondades de la especialidad.

A nivel nacional; cada año el $20 \%$ de la población adulta sufre de algún tipo de trastorno mental; y solo uno de cada 10 personas que requiere tratamiento, tiene acceso a los servicios de salud. Para garantizar la atención de salud mental de más peruanos, la Reforma de Salud ha incorporado la atención especializada en el Nivel Primario. Sin embargo, en el territorio nacional existe un aproximado de 700 psiquiatras, cantidad con la cual es difícil cubrir las necesidades de atención en los más de 7000 establecimientos de salud del país (10).

Se han atribuido de manera intuitiva determinados rasgos de personalidad para cada especialidad médica, lo cual incluye a la psiquiatría. En vista de la gran necesidad de médicos psiquiatras en el país, se requiere comprobar de forma científica la existencia de rasgos de personalidad relacionados, así como otros factores asociados a la elección de la psiquiatría como preferencia vocacional entre los estudiantes de medicina para que a partir de ellos se pueda fomentar el incremento de estudiantes interesados en la especialidad, implementando mejoras principalmente en las universidades.

Por ello, el objetivo del estudio fue determinar los factores asociados a la elección de la especialidad de psiquiatría en internos de medicina de dos universidades privadas del Perú.

\section{MATERIAL Y MÉTODOS}

Estudio observacional, transversal. Se realizó durante el año 2017, en internos de medicina humana procedentes de las universidades Ricardo Palma (URP) y San Juan Bautista (USJB) en Lima, Perú, las cuales contaron con las siguientes poblaciones: $180 \mathrm{y}$ 200 internos, respectivamente.

Para el estudio de la variable personalidad, se aplicó el Inventario de Personalidad-Eysenck forma B (EPI), validado en Perú por Anicama (11) en 1974. Este inventario mide las dimensiones de 
la personalidad: introversión-extroversión (E) y neuroticismo (estabilidad- inestabilidad) $(\mathrm{N})$. Consta de 57 ítems con respuestas dicotómicas forzadas "si" o "no". Así mismo, tiene una escala de veracidad para detectar mentiras ("L"), que permite conocer el grado de sinceridad del sujeto.

La variable percepción del proceso enseñanzaaprendizaje del curso de psiquiatría durante el pregrado fue evaluada mediante la Escala de Valoración del Proceso Enseñanza-Aprendizaje, que consta de las dimensiones: Aspecto organizativo (6 ítems), aspecto pedagógico (6 ítems) y aspecto tecnológico (6 ítems), haciendo un total de 18 enunciados. Dicho instrumento fue validado y adaptado a la población peruana en el año 2016 (12).

Se aplicó además el Cuestionario de Salud General de Goldberg (GHQ 12) para determinar el riesgo o la presencia de enfermedad mental no psicótica ni orgánico-cerebral. Consta de doce preguntas con cuatro alternativas cada una, dos de las cuales tienen un puntaje igual a cero, y las dos restantes un punto cada una. El instrumento fue validado en Perú por Contreras (13); se acepta un puntaje mayor o igual cinco (4) como de riesgo para patología emocional, condición que se denomina Goldberg positivo.

El estudio de las variables sexo y parientes con carreras afines a la salud mental se realizó por medio de la ficha de recolección de dato que se administró a cada interno de medicina junto con los instrumentos antes mencionados. Las encuestas fueron desarrolladas de forma anónima, solicitando previamente el consentimiento informado de cada unidad de estudio. Se respetó el derecho de autonomía al respetar la decisión de los internos de participar o no en el estudio.

Para el control de calidad de los datos se verificó que cada instrumento haya sido llenado de manera adecuada y en forma completa. Se descartaron los que fueron respondidos de forma parcial. En cuanto a los test de personalidad, aquellos que obtuvieron un puntaje mayor a 4 en la dimensión de verdad - mentira (L) fueron considerados como inválidos.

Se utilizó la prueba de Chi cuadrado de Pearson para determinar la existencia de asociación entre las variables. Además, se realizó análisis de regresión logística multivariada para evaluar la posible influencia de variables confusoras. Los datos fueron procesados con el Programa estadístico SPSS v. 22.0.

El estudio fue aprobado y respaldado por el Comité de ética de la Universidad Ricardo Palma.

\section{RESULTADOS}

De las 259 encuestas recolectadas, se excluyeron $36(14 \%)$ por tener un puntaje mayor a 4 en la escala de verdad - mentira (L) del Test de Personalidad de Eysenck. Se evaluaron 123 encuestas resueltas por los internos de la URP y 100 por internos de la USJB.

El rango de edades de la población total estudiada fue de 21 a 46 años, siendo el promedio de 26 años. El $59,6 \%$ de los internos de medicina humana estudiados

Tabla 1. Características de los internos de medicina humana según universidad y sexo.

\begin{tabular}{|c|c|c|c|c|c|c|}
\hline \multirow{2}{*}{ Variables } & \multicolumn{3}{|c|}{ Universidad Privada $1(n=123)$} & \multicolumn{3}{|c|}{ Universidad Privada 2 (n=100) } \\
\hline & $\mathbf{F}(\mathbf{n}=67)$ & $M(n=56)$ & Total & $F(n=67)$ & $M(n=33)$ & Total \\
\hline \multicolumn{7}{|l|}{ Eligen Psiquiatría } \\
\hline Sí & $12(17,9 \%)$ & $7(12,5 \%)$ & 19 & $9(13,4 \%)$ & $7(21,2 \%)$ & 16 \\
\hline No & $55(82,1 \%)$ & $49(87,5 \%)$ & 104 & $58(86,6 \%)$ & $26(78,8 \%)$ & 84 \\
\hline \multicolumn{7}{|c|}{ Parientes que se desempeñan en el Área de la Salud mental } \\
\hline Sí & $7(10,4 \%)$ & $5(8,9 \%)$ & 12 & $13(19,4 \%)$ & $6(18,2 \%)$ & 19 \\
\hline No & $60(89,6 \%)$ & $51(91,1 \%)$ & 111 & $54(80,6 \%)$ & $27(81,8 \%)$ & 81 \\
\hline \multicolumn{7}{|c|}{ Percepción de la enseñanza del Curso de Psiquiatría } \\
\hline Puntaje Alto & $35(52,2 \%)$ & $33(58,9 \%)$ & 68 & $34(50,7 \%)$ & $17(51,5 \%)$ & 51 \\
\hline Puntaje Bajo & $32(47,8 \%)$ & $23(41,1 \%)$ & 55 & $33(49,3 \%)$ & $16(48,5 \%)$ & 49 \\
\hline \multicolumn{7}{|l|}{ Salud Mental } \\
\hline Goldberg Positivo & $11(16,4 \%)$ & $7(12,5 \%)$ & 18 & $10(14,9 \%)$ & $6(18,2 \%)$ & 16 \\
\hline Goldberg Negativo & $56(83,6 \%)$ & $49(87,5 \%)$ & 105 & $57(85,1 \%)$ & $27(81,8 \%)$ & 84 \\
\hline
\end{tabular}


fueron de sexo femenino; además, las mujeres de ambas universidades fueron quienes, en su mayoría, escogieron psiquiatría como especialidad médica (tabla 1). En la tabla 2 se detallan las características de los internos de medicina humana que eligieron psiquiatría según universidad de procedencia; 35 internos eligieron seguir psiquiatría y aproximadamente el $60 \%$ fueron mujeres.

En el gráfico 1, se observa que alrededor del $40 \%$ del total de internos de medicina humana de ambas universidades tuvieron una personalidad sanguínea, de los cuales el 10,3\% eligieron psiquiatría como especialidad a seguir. Los resultados del Cuestionario de Salud Mental de Goldberg, mostraron que el 15,7\% tuvo riesgo de desarrollar alguna psicopatología y entre los internos que eligieron seguir psiquiatría e riesgo fue bajo (gráfico 2).

Se encontró asociación estadísticamente significativa entre la elección de psiquiatría como especialidad con la personalidad sanguínea $(\mathrm{p}=0,001)$, extroversión - introversión $(\mathrm{p}=0,02)$ y neuroticismo $(\mathrm{p}=0,003)$. Es importante mencionar que, a partir

Tabla 2. Características de los internos de medicina que eligieron psiquiatría como especialidad según universidad.

\begin{tabular}{|c|c|c|c|c|c|}
\hline \multirow{2}{*}{ Variables } & \multicolumn{2}{|c|}{$\begin{array}{c}\text { Universidad Privada } 1 \\
(\mathrm{n}=19)\end{array}$} & \multicolumn{2}{|c|}{$\begin{array}{c}\text { Universidad Privada } 2 \\
(\mathrm{n}=16)\end{array}$} & \multirow{2}{*}{$\mathbf{p}^{*}$} \\
\hline & $\mathbf{n}$ & $\%$ & n & $\%$ & \\
\hline \multicolumn{6}{|c|}{ Percepción de la enseñanza del curso de psiquiatría } \\
\hline Puntaje alto & 11 & 57,9 & 7 & 43,8 & \multirow{2}{*}{0,62} \\
\hline Puntaje Bajo & 8 & 42,1 & 9 & 56,2 & \\
\hline \multicolumn{6}{|l|}{ Sexo } \\
\hline Mujer & 12 & 63,2 & 9 & 56,2 & \multirow{2}{*}{0,87} \\
\hline Varón & 7 & 36,8 & 7 & 43,8 & \\
\hline \multicolumn{6}{|l|}{ Psicopatología } \\
\hline Goldberg Positivo & 1 & 5,3 & 4 & 25,0 & \multirow{2}{*}{0,81} \\
\hline Goldberg Negativo & 18 & 94,7 & 12 & 75,0 & \\
\hline \multicolumn{6}{|c|}{ Parientes con carreras afines al Área de la Salud Mental } \\
\hline $\mathrm{Si}$ & 3 & 15,8 & 5 & 31,2 & \multirow{2}{*}{0,17} \\
\hline No & 16 & 84,2 & 11 & 68.8 & \\
\hline
\end{tabular}

*Valor de p resultante del anális is de regresión logística multivariado

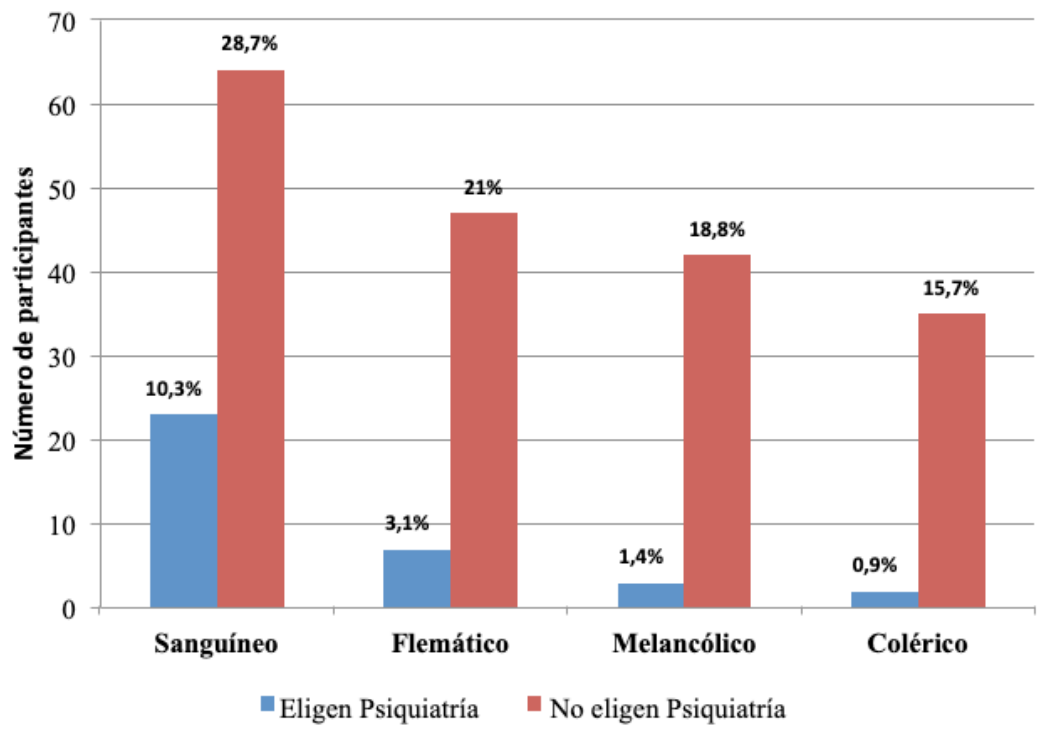

Gráfico 1. Tipos de personalidad según elección de seguir psiquiatría. 


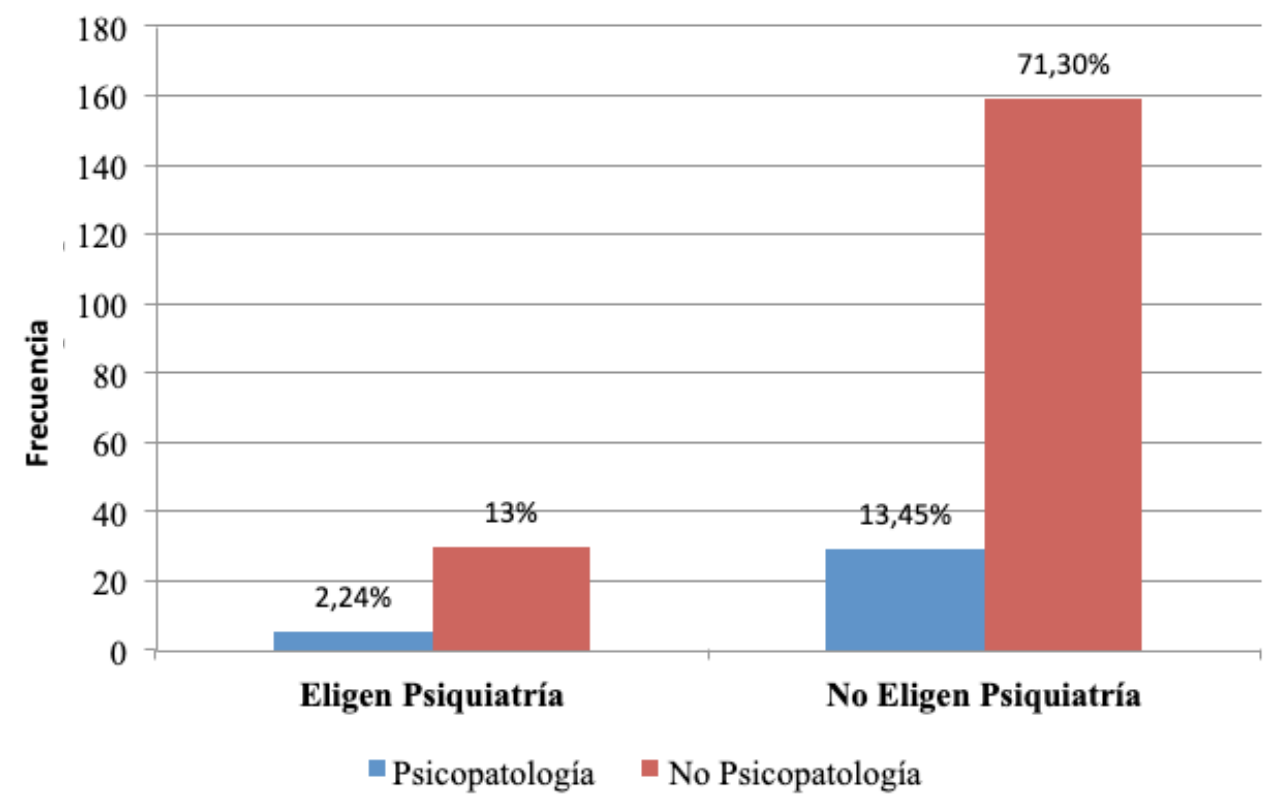

Gráfico 2. Psicopatología según elección de seguir psiquiatría.

Tabla 3. Características según elección de seguir la especialidad de Psiquiatría.

\begin{tabular}{|c|c|c|c|c|c|}
\hline \multirow[b]{2}{*}{ Variables } & \multicolumn{2}{|c|}{ SI } & \multicolumn{2}{|c|}{ NO } & \multirow{2}{*}{$\mathbf{P}^{*}$} \\
\hline & $\mathbf{n}$ & $\%$ & $\mathbf{n}$ & $\%$ & \\
\hline \multicolumn{6}{|c|}{ Percepción de la enseñanza del curso de psiquiatría } \\
\hline Puntaje alto & 18 & 51,4 & 101 & 53,7 & \multirow{2}{*}{0,62} \\
\hline Puntaje Bajo & 17 & 48,6 & 87 & 46,3 & \\
\hline \multicolumn{6}{|l|}{ Sexo } \\
\hline Mujer & 21 & 60,0 & 113 & 60,1 & \multirow{2}{*}{0,87} \\
\hline Varón & 14 & 40,0 & 75 & 39,9 & \\
\hline \multicolumn{6}{|l|}{ Psicopatología } \\
\hline Goldberg Positivo & 5 & 14,3 & 29 & 15,4 & \multirow{2}{*}{0,81} \\
\hline Goldberg Negativo & 30 & 85,7 & 159 & 84,6 & \\
\hline \multicolumn{6}{|c|}{ Parientes con carreras afines al Área de la Salud Mental } \\
\hline $\mathrm{Si}$ & 8 & 22,9 & 23 & 12,2 & \multirow{2}{*}{0,17} \\
\hline No & 27 & 77.1 & 165 & 87,8 & \\
\hline
\end{tabular}

del procesamiento de los resultados por medio de un análisis logístico multivariado, se obtuvo una razón de verosimilitud por debajo de $0,05(\mathrm{p}=0,03)$, además los valores de $\mathrm{p}$ correspondientes a cada variable se detallan en las tablas 2 y 3 .

\section{DISCUSIÓN}

Este es uno de los primeros estudios en el Perú, dirigido a determinar los factores asociados a la elección de la especialidad de Psiquiatría en Internos de Medicina, utilizando instrumentos previamente validados. En vista de la gran necesidad de médicos psiquiatras en el país, y a fin de fomentar el incremento de estudiantes interesados en tal especialidad, este estudio buscó comprobar de manera científica la existencia de rasgos de personalidad relacionados, así como otros factores asociados a la elección de la psiquiatría. 
Los resultados de este estudio evidenciaron que los internos de medicina que muestran preferencia por la especialidad de psiquiatría son extrovertidos y estables de acuerdo a las dimensiones del Test de Personalidad de Eysenck en su forma B. La personalidad tipo sanguínea fue la que con mayor frecuencia se encontró entre los internos de medicina que eligieron psiquiatría; además, demostró tener asociación significativa con la elección de esta especialidad. Por otro lado, no se encontró asociación entre la percepción del proceso de la enseñanza - aprendizaje del curso de psiquiatría, sexo, riesgo de psicopatología, y tener parientes con carreras afines al área de la salud mental con la elección de psiquiatría.

De manera semejante diferentes autores asociaron la elección de psiquiatría con determinadas características de la personalidad. Funkenstein (14), asoció la psiquiatría con una mayor extroversión y estabilidad. Por el contrario, Pasnau, Bayley (15) y Garfinkel, Waring (16), encontraron en una muestra de residentes de psiquiatría mayor inestabilidad emocional con puntuaciones elevadas en la escala de neuroticismo; y Monleon-Moscardo (5), tras estudiar a una población de 1448 estudiantes de medicina, demostró resultados semejantes. Además, Zeldow (17), asoció la elección de psiquiatría con una mayor introversión, lo cual demuestra que los resultados pueden variar dependiendo de la población.

Por otro lado, en nuestro estudio se encontró asociación significativa entre la personalidad tipo sanguínea y la elección de psiquiatría. Aunque no disponemos de estudios que aborden directamente este resultado, es importante mencionar el trabajo de Sana Jafrani $(18,19)$ quien tras aplicar el instrumento Myers Briggs Type (MBTI) en 400 estudiantes de medicina, halló asociación significativa entre la dimensión de la personalidad correspondiente a la sensibilidad - intuición con elección de psiquiatría, y puesto que la intuición y la sensibilidad son características predominantes en la personalidad sanguínea, se puede decir que es coherente con el resultado obtenido en nuestra investigación.

Aproximadamente, el $55 \%$ de los internos de medicina humana tuvieron una percepción alta respecto al proceso de enseñanza y aprendizaje del curso de psiquiatría Aunque el análisis estadístico no evidenció asociación significativa entre esta variable y la elección de psiquiatría como especialidad, es esperable que la buena enseñanza del curso influya de alguna manera en la decisión de optar especializarse en psiquiatría por lo que es importante seguir mejorando la calidad de enseñanza del curso de psiquiatría. Según esto, Perales (20), después de entrevistar a 48 psiquiatras, confirmó que los cursos de psiquiatría y psicología desarrollados de manera adecuada en el pregrado permiten definir la vocación por la psiquiatría y sus contenidos terminan por convencer al estudiante de las bondades de la especialidad, así como a dar relevancia a los aspectos no somáticos a menudo desdeñados en otras asignaturas.

En el presente estudio, el $22,9 \%$ de los internos que optarían por psiquiatría tenían familiares que se desempeñan en carreras afines al área de la salud mental, pero no se encontró asociación significativa con la elección de psiquiatría. Este resultado se puede explicar a partir de lo sostenido por Perales et al. (20), quienes afirman "en el desarrollo de la vocación psiquiátrica existe influencia de las figuras parentales, así como de otros familiares, constituyendo muchas veces una fuerza opositora para la elección de psiquiatría, quizás por no considerarla como una especialidad de éxito en la práctica médica".

No se encontró asociación entre sexo y la elección de psiquiatría. De manera parecida, MonleónMoscardó tras analizar a 1484 estudiantes de medicina de la Universidad de Valencia, demostró que existe influencia significativa del sexo en la elección de especialidad médica; sin embargo, aunque psiquiatría es una especialidad mayormente escogida por mujeres, la diferencia respecto a los varones no llega a ser estadísticamente significativa (5), lo cual refuerza lo obtenido en nuestro estudio.

Finalmente, los datos obtenidos por nuestro estudio revelan que el 15,7\% del total de internos de medicina obtuvieron puntaciones compatibles con el riesgo a desarrollar alguna psicopatología; además, no se encontró asociación significativa entre psicopatología y la elección de psiquiatría.

Aunque no se encontraron estudios que demuestren asociación entre psicopatología y la elección de psiquiatría, Galván-Molina $(21,22)$, mediante cuestionarios, escalas e inventarios identificó diferentes psicopatologías en estudiantes de medicina que afectaban al $66 \%$ del total de los estudiantes evaluados, cifra que es mayor al de la prevalecía de enfermedades neuropsiquiátricas en la población general y que va aumentando a medida que los estudiantes de medicina se van acercando al término de la carrera. Resultados semejantes obtuvo Hidalgo 
(23), quien tras evaluar a estudiantes de medicina del cuarto año de carrera; encontró que $84,5 \%$ de las mujeres y $71,4 \%$ de los varones manifestaron alguna psicopatología. Por su parte, Heinze et al. (24), agregó que "los mayores niveles de psicopatología se expresan entre los estudiantes de medicina que no alcanzan los logros académicos deseados". La prevalencia de psicopatología encontrada en este estudio no confirma lo descrito por estos autores; esto podría deberse a las particularidades de la población elegida, así como a la satisfacción de encontrarse en el nivel más alto de la etapa del pre-grado. Aunque esto es cierto, la prevalencia de psicopatología entre los internos de medicina obtenida en este estudio es equiparable a la prevalencia de enfermedades neuropsiquiátricas en el Perú, la que representa el $17,5 \%$ del total de la carga de enfermedad (25).

Los servicios de psiquiatría enfrentan una difícil disponibilidad de talento humano. La elección vocacional de las distintas especialidades médicas es un proceso complejo en la que intervienen distintos factores inherentes al alumno. Los factores estudiados pudieran tener importancia relevante en la comprensión del mecanismo de elección de psiquiatría como especialidad. De esto depende la formación de un mayor número de psiquiatras para cubrir la necesidad de salud mental que tanto se requiere no solo a nivel nacional sino también en el mundo.

Una de las limitaciones del estudio es la naturaleza y el tipo de estudio: Transversal y los posibles sesgos de información y de memoria de los encuestas. Los internos de medicina fueron evaluados de dos universidades privadas en Lima, Perú, por lo cual los resultados obtenidos no pueden ser generalizados a todos los estudiantes de medicina; sería deseable que en futuros estudios se incluya a internos de medicina de universidades estatales y de universidades privadas de diversos lugares del país. El tamaño de la muestra en nuestro estudio es representativo pero limitado, los resultados obtenidos deberán ser confirmados en estudios multicéntricos con mayor poder estadístico. Asimismo, se recomienda el desarrollo de un estudio retrospectivo en una población de médicos que estén ejerciendo la psiquiatría a fin de garantizar un mejor entendimiento de los factores que influyeron en su elección.

Otros factores que influyen en la elección de la especialidad de psiquiatría han sido descritos recientemente y deberían ser incluidos en futuros estudios tales como: características de la escuela de medicina, características del estudiante, valores del estudiante, necesidades por satisfacer, y la percepción de las características de la especialidad, que deberían ser incluidos en futuros estudios. Además se han descrito factores que influyen en la elección de una especialidad médica en general: remuneración, flexibilidad en los horarios, el prestigio que otorga una determinada especialidad médica, y la influencia de los familiares, profesores y mentores (26). Aunque se ha descrito que estos factores influyen en la elección de cualquier especialidad, estas también podrían ser específicas a la elección de psiquiatría. Posteriores estudios científicos podrían confirmarlo o negarlo.

Se concluye que en los internos de medicina, con una personalidad sanguínea caracterizada predominantemente por la extroversión y la estabilidad emocional, estuvieron asociadas significativamente a la elección de psiquiatría como especialidad médica.

\section{Declaración de financiamiento y de conflictos de interés:}

El estudio fue financiado por los autores y declaran no tener conflictos de intereses.

\section{Contribución de autoría:}

LPGCR: elaboración de protocolo, recolección, diseño metodológico, recolección de datos, procesamiento estadístico de los datos, análisis de los datos, redacción final de articulo original. LECL: diseño, procesamiento estadístico de los datos, redacción final del artículo original. JADCV: diseño, procesamiento estadístico de los datos, análisis de los datos, redacción fina del artículo original.

\section{Correspondencia:}

Lucy Cedillo Ramirez.

Jr. Los Laureles 352. Dpto. 402. Santiago de Surco. Lima - Perú.

Código Postal: 15039

Celular: 993093310.

Correo electrónico: lucycedilloramirez.tj@gmail.com

\section{REFERENCIAS BIBLIOGRÁFICAS}

1. Gutierrez-Cirlos C, Naveja J, Sánchez-Mediola M. Factores relacionados con la elección de una especialidad en medicina. Inv Ed Med. 2017; 6(23):206-214.

2. Monleón P, Rojo J, García M, Valdemoror C, y Monleón A. Factores de personalidad y atracción por 
la especialidad psiquiátrica. Actas Esp Psiquiatr 2001; 29(1):14-18.

3. Bitran M, Zúñiga D, Lafuente M, Viviani P, Mena B. Influencia de la personalidad y el estilo de aprendizaje en la elección de especialidad médica. Rev méd Chile. 2005; 133(10):1191-1199.

4. Monleón P, Rojo J, García M, Valdemoror C, y Monleón A. Factores de personalidad y atracción por la especialidad psiquiátrica. Actas Esp Psiquiatr 2001; 29(1):14-18.

5. Monleón P, Rojo J, Monleón A, García M, Alonso A y Valdemoro C. Influencia del sexo en las preferencias vocacionales y rasgos de personalidad en los estudiantes de medicina. Actas Esp Psiquiatr. 2003; 31(1):24-30.

6. Lacerda P. The general practitioner and the surgeon: sterotypes and medical specialties. Rev. Hosp Clin Facu Med S Paulo. 2004; 59(1):15-24.

7. Holm-Petersen C, Vinge S, Hansen J, Gyrd-Hansen D. The impact of contact with psychiatry on senior medical students' attitudes toward psychiatry. Acta Psychiatr Scand. 2007; 116(4):308-311.

8. Alarcon A, Castro J. Rasgos de personalidad y atracción por una especialidad médica en internos de medicina. Rev Med Carr. 2017; 4(1):37.

9. Diez-Canseco F, Ipince A, Toyama M, BenateGalvez Y, Galán-Rodas E, Medina-Verástegui JC, et al. Atendiendo la salud mental de las personas con enfermedades crónicas no transmisibles en el Perú: Retos y oportunidades para la integración de cuidados en el primer nivel de atención. Rev Peru Med Exp. Salud Publica. 2014; 31(1):131-136.

10. Perales A, Sánchez E, Mendoza A, Huamaní Ch. Vocación médica en estudiantes de medicina de una universidad peruana. An Fac med. 2014; 75(1):37-42.

11. Anicama J. Estandarización de la prueba de Eysenck en diferentes sectores poblacionales de Lima. Tesis de grado. Lima, Perú: Universidad Nacional Federico Villarreal; 1974. 91pp.

12. Blancas E. Proceso de enseñanza-aprendizaje y nivel de satisfacción de los estudiantes de la Escuela Profesional de Educación Primaria. Horizonte Ciencia. 2016; 6(10):205-217.

13. Contreras F. Principales Conductas del Estilo de Vida Asociadas a la Salud Mental de los Docentes de la Universidad Peruana Unión. Rev Inv Univ. 2013; 2(2):51-59.

14. Funkenstein DH. Factors affecting career choices of medical students. En: Shapiro E, Lowenstein L, eds. Becoming a Physician. Cambridge: Mass; 1979. p:1958-1976.

15. Pasnau RO, Bayley SJ. Personality changes in the first year of Psychiatric training. Am J Psychiatry. $1971 ; 128: 79-83$.
16. Garfinkel P, Waring EM. Personality, Interests and Emotional Disturbance in Psychiatric Residents. Am J Psychiatry. 1981; 138:51-61.

17. Zeldow PB, Daugherty SR. Personality Profiles and Speciality Choices of Students from Two Medical School Classes. Acad Med. 1991; 66:283-287.

18. Yang Ch, Richard G, Durkin M. The association between Myers-Briggs Type Indicator and Psychiatry as the specialty choice. Int J Med Educ. 2016; 7:4851.

19. Jafrani S, Zehra N, Zehra M, Abuzar S, Abubakar s, Azhar R. Assessment of personality type and medical specialty choice among medical students from Karachi using Myers-Briggs Type Indicator (MBTI) tool. J Pak Med Assoc. 2017; 67(4):520-526.

20. Perales A, Mendoza A, Sanchez E, Bravo E, Barahona L, Aguilar W. Vocación psiquiátrica. An Fac med. 2015; 76(2):167-180.

21. Galván-Molina JF, Jiménez-Capdeville ME, Hernández-Mata JM, Arellano-Cano JR. Sistema de tamizaje de psicopatología en estudiantes de Medicina. Gac Méd Méx. 2017; 153(1):75-87.

22. Loayza-Castro J, Correa-López L, Cabello-Vela C, Huamán-García M, Cedillo-Ramirez L, Vela-Ruiz M, et al. Síndrome de Burnout en Estudiantes Universitarios: Tendencias Actuales. Rev Fac Med Hum. 2016; 16(1):31-36.

23. Motta RL, Hidalgo PM, Gola V, Ortiz P, Miatello $\mathrm{R}$. Trastorno de ansiedad y depresión en una muestra de estudiantes de medicina. Salud mental, psicología y psicopatología del niño, adolescente y su familia. Curso virtual interdisciplinario a distancia. 2017: 1-14. Disponible en: https://psicoadolescencia.com. ar/docs/3/final6.pdf

24. Heinze G, Vargas BE, Cortés Sotres JF. Síntomas psiquiátricos y rasgos de personalidad en dos grupos opuestos de la Facultad de Medicina de la UNAM. Salud Mental. 2008; 31:343-350.

25. Ministerio de Salud. Dirección general de intervenciones estratégicas en salud pública. Lineamientos de política sectorial en salud mental. Dirección de salud mental. Lima, Perú; 2018: 12-14.

26. Yang Y, Li J, Wu X, et al. Factors influencing subspecialty choice among medical students: a systematic review and meta-analysis. BMJ Open. 2019; 9(3):7-9.

Recibido: 11/02/2019

Aceptado: 09/07/2019 